\title{
Evaluation of Motor-Assisted Gloves (SEM Glove) for Patients with Functional Finger Disorders: A Clinical Pilot Study
}

\author{
RYUKI HASHIDA***, HIROO MATSUSE*,**, MASAFUMI BEKKI*,**, MASAYUKI OMOTO*,**, \\ SHIMPEI MORIMOTO ${ }^{\dagger}$, TOMOKO HINO*, YUUJI HARANO*, CHIKAHIRO IWASA*, \\ KAZUKI MIYAMOTO*, MASAKUNI HARAGUCHI*, TAKESHI NAGO*,** \\ AND NAOTO SHIBA**** \\ *Division of Rehabilitation, Kurume University Hospital, \\ **Department of Orthopedics, Kurume University School of Medicine, Kurume 830-0011, Japan, \\ ${ }^{\dagger}$ Innovation Platform \& Office for Precision Medicine, Graduate School of Biomedical Sciences, \\ Nagasaki University, Nagasaki 852-8501 Japan \\ Received 30 January 2018, accepted 29 June 2018 \\ J-STAGE advance publication 11 March 2019
}

Edited by KIMIAKI SATO

\begin{abstract}
Summary: The SEM Glove developed by Bioservo Technologies AB is a new device that increases grip and pinch force. The purpose of this study was to evaluate the effectiveness of the device on the grip and pinch strength of patients with functional disorders of the fingers.

Materials and Method: 30 hospitalized patients with upper limb functional disorder were enrolled. The assistance of the device for the grip and pinch strength of each subject were assessed by the difference between the measured values with and without the SEM Glove. The 95\% confidence interval of the difference was calculated across the subjects, and statistical significance was defined as when the lower limit was a positive value (corresponding with a paired t-test at a significance level of 0.05). The odds ratio was calculated in the study of subject adaptation, with statistical significance set using Fisher's exact test at a significance level of 0.05 .

Results: Grip strength significantly decreased (worn-not worn difference $(\mathrm{kg})$ : mean $=-3.7$, CI95 $(-5.4,-2.1)$ ). Pinch strength (thumb - middle finger) significantly increased (worn-not worn difference $(\mathrm{N})$ : mean $=-4.1, \mathrm{CI} 95$ $(1.6,6.6))$. Analysis of factors related to improvement in hand function when wearing the SEM Glove extracted manual muscle tests (MMTs) of the upper extremity 4 or higher. The odds ratio was 6.11 .

Conclusions: Use of the SEM Glove improved the pinch strength of patients with functional disorders of the hands.
\end{abstract}

Key words pinch strength, hand function, upper limb disorders

\section{INTRODUCTION}

The annual incidence of stroke is in the USA 795,000, and the annual incidence of traumatic brain injury is 1.7 million $[1,2]$. With an expected increase in the aged population during the next decade, we can foresee an increased number of stroke and traumatic head and neck injuries. Stroke, head, and neck injuries leave the affected person with various sensory, motor, and/or autonomic defects $[3,4]$. These diseases affect the activities of daily living as well as occupational activities later in life. Upper limb and hand function plays an important role in our daily life. In particular, functional disorders of the hands are directly linked to the

Corresponding Author: Ryuki Hashida, M.D., Ph.D., Department of Orthopedics, Kurume University School of Medicine, 67 Asahi-machi, Kurume 8300011, Japan. Tel: +81-942-31-7568,Fax: +81-942-35-0709, E-mail: hashida_ryuuki@med.kurume-u.ac.jp 
deterioration of Activities of Daily Living (ADL) and a decrease in work capacity [5]. Various apparatuses and artificial limbs have been developed over the years for the purpose of compensating for lost function.

Currently, a number of devices that apply electrical stimulation are being put to clinical use $[6,7]$. Many clinical studies have been conducted that report the use of Functional Electrical Stimulation (FES). FES is defined as the application of neuromuscular electrical stimulation to facilitate purposeful tasks where normal neural function has been damaged or destroyed, e.g. grasping an object or assisting with walking. The use of FES devices has been reported to improve upper limb function after spinal cord injury or stroke [8]. FES can be applied using surface, percutaneous, or implanted electrodes. In particular, the use of surface electrodes is a simple and useful method to compensate for lost function; however, the electrodes need to be placed directly over the motor points of the muscle. The improper placement of an electrode can affect comfort levels and muscle contraction strength. Moreover, electrical stimulation can be uncomfortable [6]. Although FES is effective for function recovery, there are numerous disadvantages.

The SEM Glove developed by Bioservo Technologies $\mathrm{AB}$ (Sweden) [9] is a new device that complements hand function. It is essentially a hand orthotic device that detects the intention to grip and manipulate an object, and then applies a proportional compensation to assist with the intended task. The SEM Glove increases the grip and pinch force of patients with functional disorders of the fingers and prolongs their grasping duration. When the wearer begins to grip an object, information from the tactile sensors determines how much additional force the wearer needs to grip the object and the glove 'strengthens' the hand accordingly. By wearing this device, compensation is made for reduced hand function, with the expectation that this will lead to improvement in the Quality of Life. Nilsson et al. showed that the SEM glove improved the motor performance of chronic neuromuscular disease patients (i.e. post-polio syndrome) [9]. The results showed that the SEM glove can provide orthotic benefits while remaining compact and light without a bulky exoskeleton. However, patients with hand function disorders caused by other diseases, such as cranial nerve diseases, or orthopedic diseases have different needs and degrees of disorder, thus it is necessary to adapt the SEM glove for each clinical situation.

The purpose of this study was to evaluate the effectiveness of the SEM glove on grip and pinch strength related to upper limb and hand function disturbance.

\section{METHODS}

\section{Ethics}

The study protocol conformed to the ethical guidelines of the Declaration of Helsinki as reflected in the prior approval given by the institutional review board of Kurume University (Kurume, Japan). Informed consent for participation in the study was obtained from each patient (approval ID:14155) UMIN ID (UMIN000026389).

\section{Mechanical Design of the SEM Glove}

Developed by Bioservo in Sweden, the SEM Glove stands for "Soft Extra Muscles Glove" and has been promoted in a collaborative research effort with the Royal Institute of Technology of Sweden. The SEM Glove was developed to increase the grip and pinch force of patients with functional disorders of the fingers and to prolong their grasping duration, as described in detail elsewhere [10]. Briefly, fine grasping force is detected by pressure sensors located at the finger-pulp regions of the thumb, middle finger, and ring finger, and finger flexion is induced by the operation of a motor located in the control pack that pulls the built-in wires, allowing grasping movements to be motor-assisted (Fig. 1a,b). This device can be easily worn like an ordinary glove, and the control pack is designed to be lightweight, at approximately $500 \mathrm{~g}$, making it portable (Fig. 1c). The grip strength and sensitivity of the fingertip sensors can be set according to the intended use.

\section{Subjects}

Inclusion criteria included hospitalized patients who were (i) 20 years of age or more, (ii) had upper limb functional disorder, and (iii) had no defects of the fingers. Exclusion criteria were patients with (i) dermatosis that did not allow the wearing of a glove, (ii) blood pressure at rest more than $160 / 100 \mathrm{mmHg}$, and (iii) complications that cause difficulty in performing rehabilitation. Final enrollment was 30 hospitalized patients with upper limb functional disorder admitted to Kurume University hospital from May 17, 2015 to February 25, 2016.

\section{Patient classification and testing}

Manual muscle test (MMT) and Brunnstrom Recovery Stages (Brs) were used for classification of the patients by height, weight, and functional disorder of the upper limb.

Moreover, we conducted the following evaluations with subjects wearing or not wearing the SEM glove. 


\section{Grip strength}

Testing was performed with participants in the standing position with the elbow in full extension and the forearm in the neutral position. The best record of two attempts of maximal voluntary contraction using the diseased limb was considered for analyses.

\section{Pinch strength}

Pinch strength was measured with the Isoforce GT-300 (OG Wellness, 1835-7 Miyoshi, Naka-ku, Okayama city, Okayama prefecture, Japan). The best record of two attempts of maximal voluntary contraction using the diseased middle finger with the patient in the sitting position was considered for analysis.

\section{Action Research Arm Test (ARAT)}

The Action Research Arm Test (ARAT) has been shown to be a reliable, valid measure of limb motor status after stroke [11]. Upper limb function was evaluated by use of the ARAT (using the "grasping movement" subtest alone in consideration of the design concept of the SEM Glove).

\section{Activities of Daily Living (ADL)}

Patients were asked to describe their recovery status using the following evaluation tools: $100-\mathrm{mm}$ vis- ual analogue scale (VAS). The 100-mm VAS is an assessment of recovery, where one end of the scale is 0 and the other end is 100 , representing the worst and the best quality of recovery, respectively. We used the following methods to investigate the effect on ADL of wearing the SEM glove: (i) interviewing examinees about difficulties or problems in their daily life caused by upper limb functional disorders; (ii) evaluations by VAS were conducted regarding the "level of satisfaction with daily life movements" before wearing the SEM Glove; and (iii) the "degree of improvement in daily life movements " when wearing the SEM Glove.

\section{Statistical analysis}

The assistance of the SEM Glove to the grip and pinch strength was assessed for each subject by the difference between the measured values with and without the SEM Glove ("improvement value"). The 95\% confidence interval of the difference (CI95 (lower limit, upper limit)) was calculated across the subjects, and statistical significance was defined as when 0 was not included within the interval (corresponding with a two-tailed paired $t$ test at a significance level of 0.05).

Next, in order to judge the adaptation of subjects to the SEM Glove, we did the following analysis. The SEM Glove complements only hand function, therefore we hypothesized that upper limb strength and mo- a

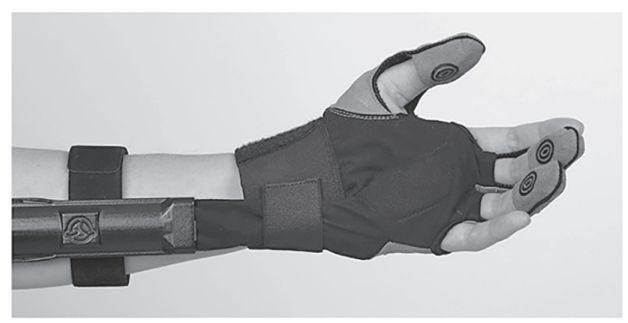

b

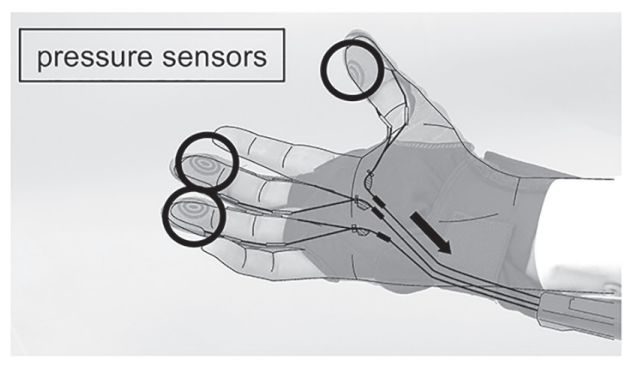

C

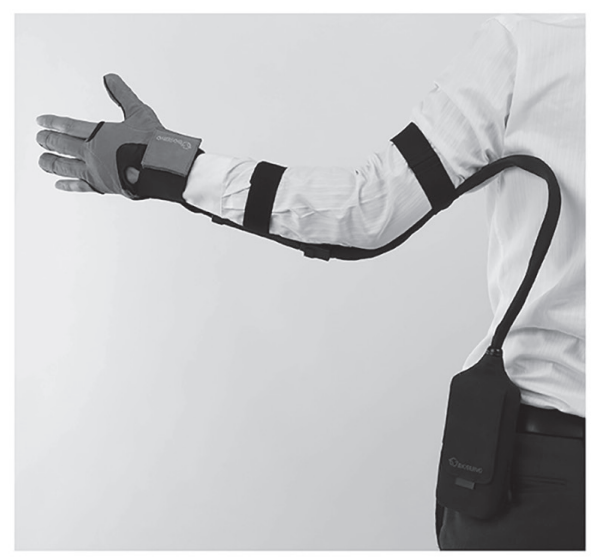

Fig. 1. The SEM Glove

The device used in this study is a compensatory assistive device, the Soft Extra Muscle (SEM) Glove. The glove detects the grip of an object and 'strengthens' the hand to generate adequate grip force and the sensitivity of the grip. 
tor function was needed to receive adequate assistance from the SEM glove. Thus, we defined the subject's adaptability ("adaptable" or "not adaptable") by arm and hand Brs (each stage 5 or more) and shoulder flexion, elbow extension, wrist dorsiflexion strength (each of strength MMT 4 or more). Then we estimated the effect of adaptability (defined above) on the odds of achieving "hand-function improvement", which was measured by ARAT or pinch strength (for patients who obtained a full score in ARAT both with and without the SEM glove). Fisher's exact test at a level of 0.05 was conducted to determine the statistical significance of the odds ratio.

SAS 9.3 and R ver.3.4.3 was used as the statistical software.

Clinical monitoring, data management and statistical analyses were undertaken by an independent contract research organization (IBERICA Co., Ltd., Fukuoka, Japan).

\section{RESULTS}

Thirty subjects participated in this study, without any injury. While wearing the SEM glove, no subjects complained of discomfort or pain. Patient characteristics are summarized in Table 1 . The average age was 62.7 years, and the ratio of women to men was $1: 1$ $(15 / 15)$. Of the 30 subjects, 10 had cranial nerve diseases, 17 orthopedic diseases, and 3 other diseases (Table 2).

\section{Grip strength}

Improvement in grip strength was seen for only 5 of the 30 subjects when wearing the SEM Glove, giving a statistically significant decrease in grip strength. [improvement value $(\mathrm{kg})$ : mean $=-3.7$, CI95 $(-5.4$, -2.1)] (Fig. 2a).

An additional analysis divided the subjects by the arm evaluated (left or right) as shown in Fig. 2b [left: $\mathrm{N}=13$, mean $=-5.0 \mathrm{~kg}, \mathrm{CI} 95(-7.3,-2.7) /$ right: $\mathrm{N}=$ 11 , mean $=-2.8 \mathrm{~kg}, \mathrm{CI} 95(-4.6,-1.0)]$. The affected side was not available for six subjects.

\section{Pinch strength}

Pinch strength (thumb - middle finger) improved in 20 of the 30 subjects when wearing the SEM Glove. The average improvement value across the all subjects

TABLE 1.

Patient Characteristics

\begin{tabular}{ccc}
\hline & $\operatorname{mean}(\mathrm{SD})$ & Range(min-max) \\
\hline Age & $62.7(16.0)$ & $21.0-81.0$ \\
Height & $159.8(10.1)$ & $137.8-175.0$ \\
Weight & $59.7(12.5)$ & $37.1-88.2$ \\
Sex female/male & $15 / 15$ & \\
MMT shoulder flexure & $3.9(0.9)$ & $3-5$ \\
MMT elbow extension & $4.1(0.6)$ & $1-5$ \\
MMT wrist dorsiflexion & $3.9(1.0)$ & $4-6$ \\
Brs- arm & $4.7(0.8)$ & $4-6$ \\
Brs- hand & $4.8(1.0)$ &
\end{tabular}

All data expressed as mean(SD).

The Brs data includes only six cases of cranial nerve disease.

Brs, Brunnstrom recovery stage; MMT, manual muscle test.

TABLE 2

Target diseases

\begin{tabular}{lll} 
Cranial nerve diseases $(\mathrm{N}=10)$ & Orthopedic diseases $(\mathrm{N}=17)$ & Other diseases $(\mathrm{N}=3)$ \\
\hline Cerebral stroke: 6 cases & Cervical spondylosis: 7 cases & Pericarditis \\
Brain tumor: 2 cases & Spinal cord injury: 2 cases & After left breast cancer surgery \\
Spinal cord tumor: 2 cases & Finger and forearm injury: 4 cases & Muscular dystrophy \\
& After carpal tunnel surgery: 2 cases & \\
& Articular rheumatism: 2 cases & \\
\hline
\end{tabular}


was significant [improvement value $(\mathrm{N})$ : mean $=4.1$, CI95 (1.6, 6.6)] (Fig. 2a).

As in the analysis of Grip strength, we did a separate analysis of the arm tested. The improvement value was significant for the right arm $[\mathrm{N}=11$, mean $=2.7$, CI95 $(1.0,4.4)]$, but not for the left $[\mathrm{N}=13$, mean =
3.2, CI95 (- 0.7, 7.1)] (Fig. 2b).

\section{ARAT}

Perfect scores were attained by 18 subjects both when the SEM Glove was worn and when it was not worn. For the remaining 12 subjects, comparisons were a

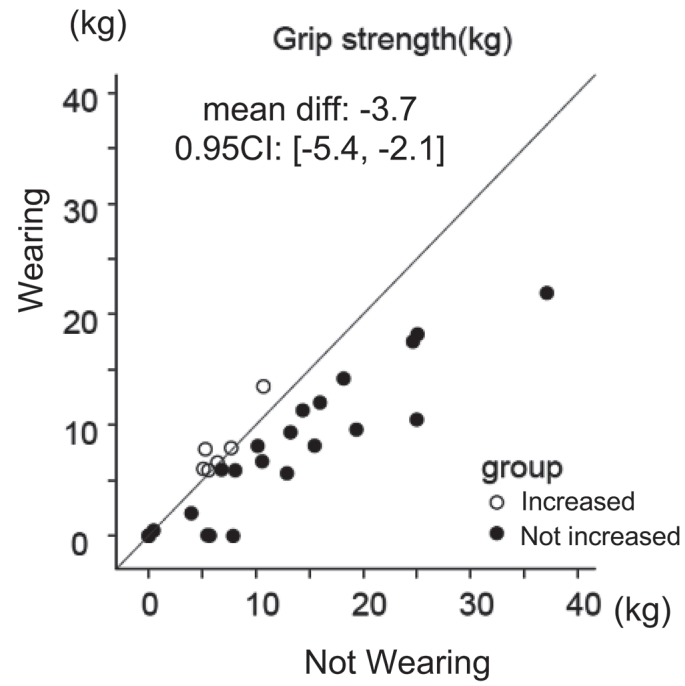

(N) Pinch strength(thumb-middle finger)

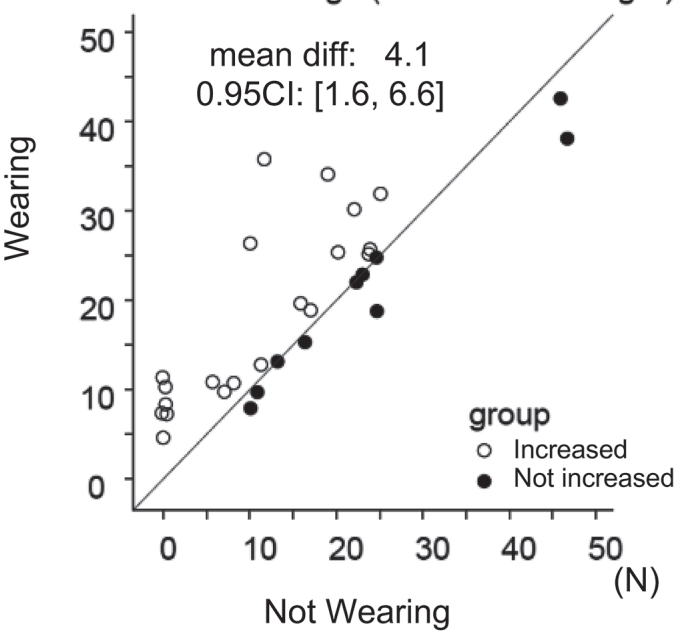

b

Increased

- Not increased

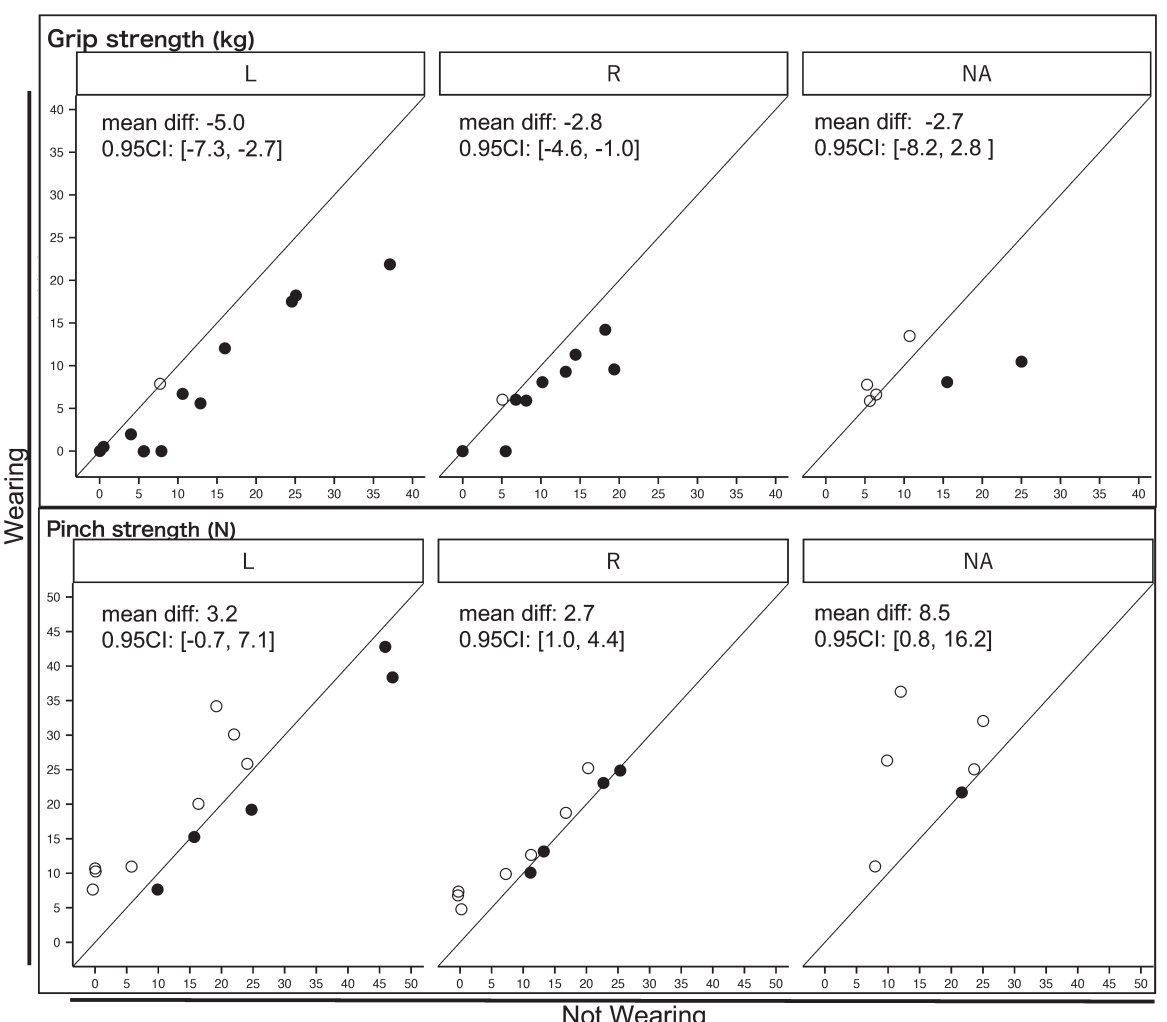

Fig. 2. Effectiveness of the SEM Glove

Vertical axis, wearing the SEM Glove: Horizontal axis, not wearing the SEM Glove (Fig. 2a). In an additional analysis, data was divided into use on the left or right arm (Fig. 2b). Data for which the measured arm is unknown is expressed as NA (not available). 
made when the SEM Glove was worn and when it was not worn, with the following results: 5 improved, 3 had no change, and 4 deteriorated.

\section{Activities of Daily Living (ADL)}

Subjects answered that improvement was made in 61 of the 80 items $(67.8 \%)$ when wearing the SEM Glove compared with not wearing the device, with 9 items $(10.0 \%)$ regarded as having no change and 20 items $(22.2 \%)$ indicating a deteriorated condition.

\section{Investigation of adaptation}

Sixteen of the thirty subjects showed improved hand function when wearing the SEM glove. No subjects for whom Brs was less than 5 showed improvement of hand function. Ten of the thirteen subjects for whom MMT (shoulder joint flexion, elbow joint extension, hand joint dorsiflexion) was 4 or higher showed improvement of hand function. The odd's ratio of adaptability for the achievement of hand-function improvement was $6.11(\mathrm{P}<0.05)$. "Adaptability" and "hand-function improvement" were defined in the "statistical analysis" section and are illustrated in Figure 3.

\section{DISCUSSION}

The SEM Glove was tested in 30 hospitalized patients at Kurume University Hospital. No joint pain or other adverse events were observed while wearing the SEM glove. The SEM Glove's safety when in use is considered to be satisfactory. Moreover, this study showed that the SEM glove improved pinch strength, which indicates improvement in hand function. Among patients with upper limb dysfunction whose upper limb MMT was 4 or more and Brunnstrom stage was 5 or more, use of the SEM glove resulted in improvement of the hand function.

By wearing an SEM glove, the pinch strength greatly increased (Fig. 2). When patients grasped a relatively large, smooth shaped object, such as when grasping a glass, the SEM glove was useful because the shape of the cup allowed a proper fit for the sensors. In contrast, when subjects grasped an unevenly formed object, there was an improper fit between the sensor and the object, which did not allow assistance to be activated because the sensor and the object did not come into full contact. This was also true for grip strength (Fig. 2). To insure the consistency of our study design,

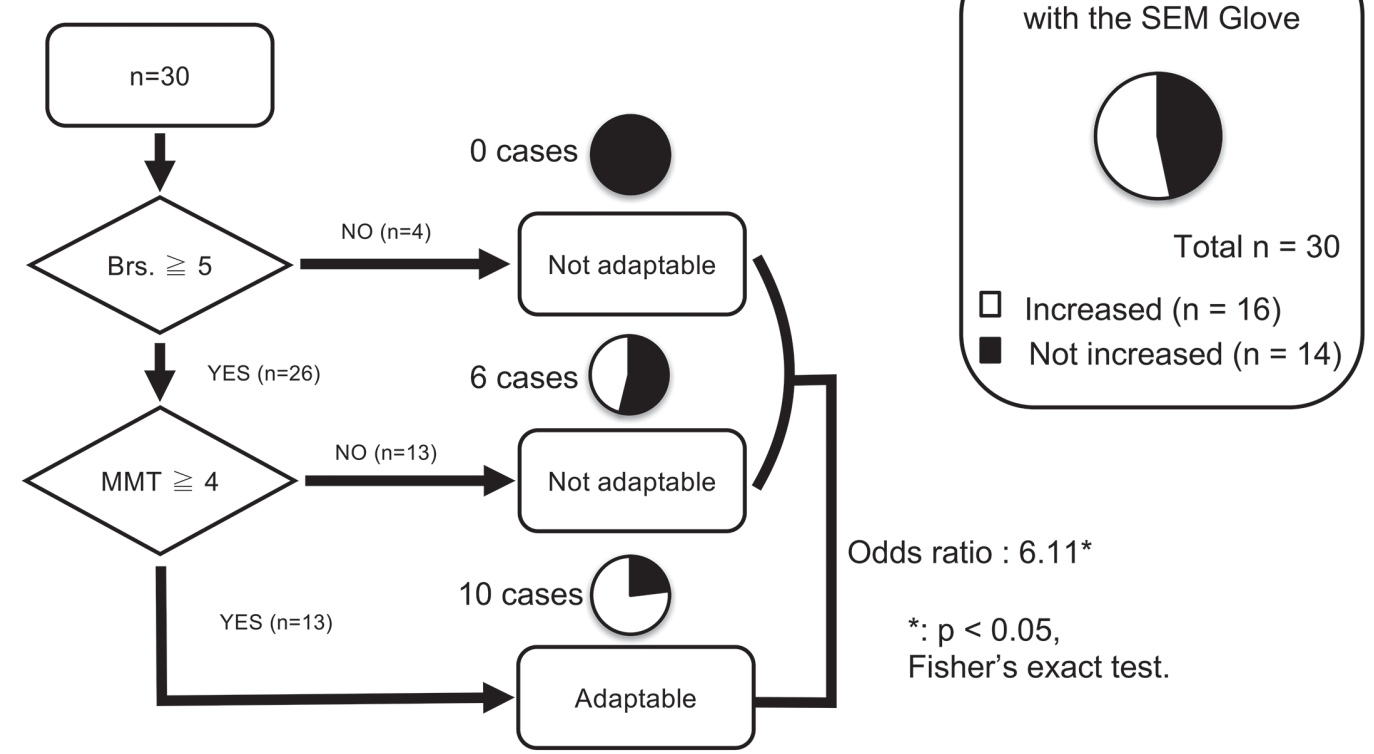

Fig. 3. Adaptation to the SEM Glove

The subjects were divided in two groups (Adaptable/ Not adaptable) by the results of upper limb strength and motor function testing. The cutoff values were set for function by Brs (both of the arm and hand) stage 5 or more and for upper limb strength by MMT (shoulder flexion, elbow extension, and wrist dorsiflexion strength) 4 or more. ARAT was applied as a hand function evaluation item. Pinch strength was alternatively taken as a hand function evaluation item for examinees with a perfect ARAT score, regardless of whether or not the SEM Glove was worn. 
the evaluation was done without changing the sensor position. The glove could easily have been modified according to the patient's disorder and hand shape and size, which would likely have resulted in better results. It will be necessary to do future study of the effectiveness of the SEM glove with adjusted sensor positions.

The purpose of this study was to investigate what kind of disabilities the device is adaptable for. When compensating for reduced hand function using the SEM glove, the upper limb's voluntary functioning is preserved because the SEM glove improves strength but not voluntariness. We attempted to determine if the SEM Glove would be effective for subjects whose upper limb muscle strength and motor function is maintained to some degree but who do not have good hand function in order to determine which patients would benefit from the assistance of the SEM Glove, as in Fig. 3. We showed that the SEM Glove would provide a benefit to subjects with deteriorated upper limb muscle strength, in particularly in the proximal muscle. For patients with muscular dystrophy or articular rheumatism whose distal upper limb strength had deteriorated, although the use of the SEM glove only improved pinch strength, the benefit conferred by the gloves was important to improving these patients' activity of daily life and quality of life.

Various apparatuses have been developed to compensate for various disorders. FES for patients with motor function impairment of the upper limb has been employed as a treatment option for many years [12]. FES is an effective rehabilitation method for various aspects of motor impairment, however, some important problems with FES remain to be resolved (e.g., muscle fatigue, habituation, etc.) [13]. Moreover, a recent review by the Cochrane group reported that there is no statistical evidence that electrical stimulation is superior to conventional physical therapy on ADL [14]. The SEM glove has some advantages as compared with FES. First, the patient can adjust the power of the SEM glove by themselves by use of the control unit. FES needs to have the stimulator device set up for the patient, necessitating one-to-one attention by a therapist [7]. Second, patients can use the SEM glove for long periods of time without muscle fatigue, whereas FES causes the rapid development of muscle fatigue in daily use because multiple motor units are stimulated synchronously in a non-selective order [12]. In the SEM glove, artificial tendons are connected to actuators in the form of electrical motors, which create the required pulling forces. In these respects, the SEM glove has the potential to take the place of conventional apparati. However, the SEM glove cannot control indi- vidual fingers or joints as finely as FES and thus will require future improvements to reach its potential.

Limitations of the study include that patients were restricted to only one test session. ARAT as used in this study is influenced by whole upper limb function. Therefore, for future study we need to choose another evaluation method that presents mainly hand function. The study design did not allow repeated training sessions with the SEM glove. Repeated training would enhance the utility in the subgroup of patients with the most potential. This study could not show whether or not the SEM glove would be effective over the long term; thus, studies using the SEM glove for a longer period of time will be necessary to evaluate its potential effectiveness. Furthermore, this study is not a RCT. An RCT comparing SEM with other conventional apparatuses will be necessary in order to show the possible superiority of the SEM system. Another limitation of the current study is the small sample size. Investigating the effectiveness of the SEM glove for cranial nerve diseases and orthopedic diseases in a study with a larger sample size would better show who would benefit the most from the SEM glove. However, this study is useful as a pilot study because it gives us information about the adaptation and effectiveness of the SEM glove.

\section{CONCLUSIONS}

We showed that the SEM Glove can improve the pinch strength of patients with functional disorders of the hands and that it improves ADL. In addition, the SEM Glove was shown to be effective in patients who had upper limb function deterioration, particularly when the patient's upper limb MMT was 4 or more and the patient was Brunnstrom stage 5 or more. The SEM glove was shown to provide good support for the hand function of patients with upper limb disorders.

FINANCIAL SUPPORT: The authors declare funding by SK-Electronics CO., LTD. These funds were used primarily for the purchase of the device. No staff members of SK-Electronics Corporation were in involved in this study or in the analysis of the results.

AUTHORS' CONTRIBUTIONS: Hashida R, Matsuse H, and Morimoto $\mathrm{S}$ participated in study conception and design, interpretation of data, and drafting of the manuscript. Bekki M, Omoto M, Hino T, Harano Y, Iwasa C, Miyamoto K, Haraguchi $\mathrm{M}$, and Nago $\mathrm{T}$ performed the experiments. Shiba $\mathrm{N}$ participated in critical revision.

\section{REFERENCES}

1. Go AS, Mozaffarian D, Roger VL, Benjamin EJ, Berry JD et 
al. Heart disease and stroke statistics--2013 update: a report from the American Heart Association. Circulation 2013; 127(1):e6-e245.

2. Kumar A and Loane DJ. Neuroinflammation after traumatic brain injury: opportunities for therapeutic intervention. Brain Behav Immun 2012; 26(8):1191-1201.

3. French JA, Rose CG, and O’Malley MK. System Characterization of MAHI EXO-II: A Robotic Exoskeleton for Upper Extremity Rehabilitation. Proc ASME Dyn Syst Control Conf 2014; 2014.

4. Watanabe M, Sakai D, Yamamoto Y, Nagai T, and Sato M, Mochida J. Analysis of predisposing factors in elderly people with type II odontoid fracture. Spine J 2014; 14(6):861866.

5. Eraifej J, Clark W, France B, Desando S, and Moore D. Effectiveness of upper limb functional electrical stimulation after stroke for the improvement of activities of daily living and motor function: a systematic review and meta-analysis. Syst Rev 2017; 6(1):40.

6. Popovic DB, Sinkaer T, Popovic MB. Electrical stimulation as a means for achieving recovery of function in stroke patients. NeuroRehabilitation 2009; 25(1):45-58.

7. Yamaguchi T, Tanabe S, Muraoka Y, Imai S, Masakado Y et al. Effects of integrated volitional control electrical stimulation (IVES) on upper extremity function in chronic stroke. Keio J Med 2011; 60(3):90-95.
8. Prochazka A, Gauthier M, Wieler M, and Kenwell Z. The bionic glove: an electrical stimulator garment that provides controlled grasp and hand opening in quadriplegia. Arch Phys Med Rehabil 1997; 78(6):608-614.

9. Nilsson M. A Helping Hand - On Innovations for Rehabilitation and Assistive Technology. Division of Neuronic Engineering School of Technology and Health KTH - Royal Institute of Technology. 2013.

10. Mats Nilsson, Johan Ingvast, Jan Wikander, and Holst Hv. The Soft Extra Muscle System for Improving the Grasping Capability in Neurological Rehabilitation. IEEE-EMBS International Conference on Biomedical Engineering and Sciences. 2012.

11. Yozbatiran N, Der-Yeghiaian L, and Cramer SC. A standardized approach to performing the action research arm test. Neurorehabil Neural Repair 2008; 22(1):78-90.

12. Quandt F and Hummel FC. The influence of functional electrical stimulation on hand motor recovery in stroke patients: a review. Exp Transl Stroke Med 2014; 6:9.

13. Popovic DB. Advances in functional electrical stimulation (FES). J Electromyogr Kinesiol 2014; 24(6):795-802.

14. Pomeroy VM, King L, Pollock A, Baily-Hallam A, and Langhorne P. Electrostimulation for promoting recovery of movement or functional ability after stroke. Cochrane Database Syst Rev 2006(2): CD003241. 